\title{
The Results Of A Longitudinal Study Of The Effects Of Network Delays On Learning
}

Jay Sullivan, Virginia Military Institute, USA Francis Bush, Virginia Military Institute, USA James Squire, Virginia Military Institute, USA Vonda Walsh, Virginia Military Institute, USA

\begin{abstract}
The use of interactive web-based teaching materials has become an indelible feature of the educational landscape over the last decade especially for technical subjects such as engineering and mathematics. While web-based simulations present great opportunity to provide students with the feedback needed for the acquisition of new concepts, it has been demonstrated that even small network delays can adversely affect the pedagogical efficacy of these simulations. In the present work a longitudinal study is performed to examine the effects of network delay on learning as student's progress from freshmen to senior year. Findings indicate that by the time students have progressed to their senior year, they have taken on a more conservative learning style and that their learning style is less sensitive to network delays than it was when they were freshmen. The study suggests that the accumulation of experience is an important factor in mitigating the effects of network delay on learning.
\end{abstract}

Keywords: Enjoyment; Feedback Delay; Latency; Learning Styles; Network Delay; Objective Comprehension; Pedagogical Efficacy; Self-Reported Comprehension

\section{INTRODUCTION}

nteractive web-based learning tools such as engineering simulations and mathematics tutorials have become increasingly popular resources for undergraduate education, rivaling even the importance of textbooks in recent years, Reisel (2010), Dollar, (2007). Beyond the walls of conventional classrooms, web-based educational software has moved on-line courses into the main stream with $61 \%$ of 2 year and 4 year educational institutions offering on-line courses as of the 2006-2007 academic year, Parsad (2008). The Sloan Consortium Annual Report states that 3.9 million students were taking on-line courses in 2007, Allen (2008), and that by the fall semester of 2010 this number had risen to 6.1 million, Allen (2011). While the ranks of students involved in some form of web based education expands every year, the effects of the physical limitations of the internet, such as bandwidth / network delay, on learning is only partially understood.

Four years ago, during the 2007-2008 academic year the authors began a systematic study on this topic using a web-based Fourier synthesis tutorial with an initial sample of 281 students to characterize the effects of network delay on learning, Squire (2008), Walsh (2011), Bush (2008). Students were provided with one of eight possible versions of an interactive tutorial, each of which was coded with a particular delay. Students were then presented with a set of conceptual questions about Fourier synthesis and instructed to use their version of the tutorial interactively to answer the questions. Students were also queried as to their enjoyment of the learning experience, as well as how confident they were about their answers to the conceptual questions. Based on this data, an initial assessment as to the effect of network delays on objective learning scores, self-assessed learning and enjoyment were made. In general, it was found that the group as a whole was fairly intolerant to delay for objective learning, with scores falling off at delays of $60 \mathrm{~ms}$; the smallest non-zero delay in the study. In contrast to the objective learning results, enjoyment declined gradually with increasing delays, and self-assessed learning ratings only decreased for delays greater than $300 \mathrm{~ms}$. 
The purpose of this paper is to report on the outcomes of a longitudinal study started in the fall semester of 2008 with a new set of students consisting of 38 freshmen. Each participant was randomly assigned a version of the Fourier synthesis tutorial and asked to use the tutorial to answer the same questions posed in the original study. This group of 38 students was reintroduced to the Fourier synthesis tutorial as seniors, with each student receiving the identical version of the tutorial that they used as freshmen. As in the original study, students' performance on the Fourier synthesis tutorial was assessed using measures of objective learning, self assessed learning and enjoyment. In addition to examining the effect of delay time on these measures, the notion of a self-critique measure is introduced as a means to gage changes in the learning characteristics of the students over time.

\section{Results of Previous Work}

The original study took place in the fall semester of 2007 and included 281 students from four different universities. 155 students identified their major as engineering or sciences, 96 stated their major as humanities and 30 did not specify a single major. The age range of participants was 15-25 years of age, with a mean age of 19.15 years, and $86 \%$ of the students were male.

A C\# interactive software application containing a hidden delay was created for the experiment, with eight different versions corresponding to delays of $0 \mathrm{~ms}$ to $420 \mathrm{~ms}$ in increments of $60 \mathrm{~ms}$. The program can be downloaded at: http://www4.vmi.edu/Faculty/squirejc/Research/Fourier_Synthesis/Fourier_Synthesis.htm

Students were randomly assigned a version of the Fourier synthesis tutorial and then were given a questionnaire to work on interactively with the tutorial program. The questionnaire consisted of 6 personal information questions to establish information about the students such as their age, gender and academic major, as well as 11 questions concerning Fourier analysis concepts and two questions related to students' enjoyment of the tutorial, and their confidence in the answers they gave. Data from questionnaires was tabulated using a Matlab program and used to calculate three measures of learning with the interactive tutorial:

- $\quad$ Objective learning: The proportion of correct answers from the 11 conceptual questions.

- $\quad$ Self Assessed Learning: How well the students felt that they performed on the 11 conceptual questions. Scoring was based on Likert scale where 5 corresponded to "very confident" in their responses and 1 corresponded to "not sure at all".

- $\quad$ Self Assessed Enjoyment: Scoring was based on a Likert scale with 5 representing the most enjoyment and 1 the least enjoyment.

In each case the mean values of these measures were calculated and plotted against the delay time, (see figures 1-3). The error bars on each of the plots are given as $+/$ - the standard deviation of the measurements. Bilinear models were fit to the data to identify the "elbows" of the various learning measures as a function of delay. In general objective learning scores were the most sensitive to network delays with a $10 \%$ drop for delays $60 \mathrm{~ms}$ or higher. Self Assessed learning, (i.e. student confidence), was much less sensitive to delay, dropping steeply only for delays greater than $300 \mathrm{~ms}$. Enjoyment on the other hand, decreases steadily from a high value of approximately 4.75 at $0 \mathrm{~ms}$ delay, until it levels off at a value near 3.7 at a delay of $300 \mathrm{~ms}$. These results are interesting in that they point out that students may be somewhat confident about their answers and happy with the learning experience at delays which produce poor objective learning results. The study concluded that delay times must be minimized to meet objective learning goals. 


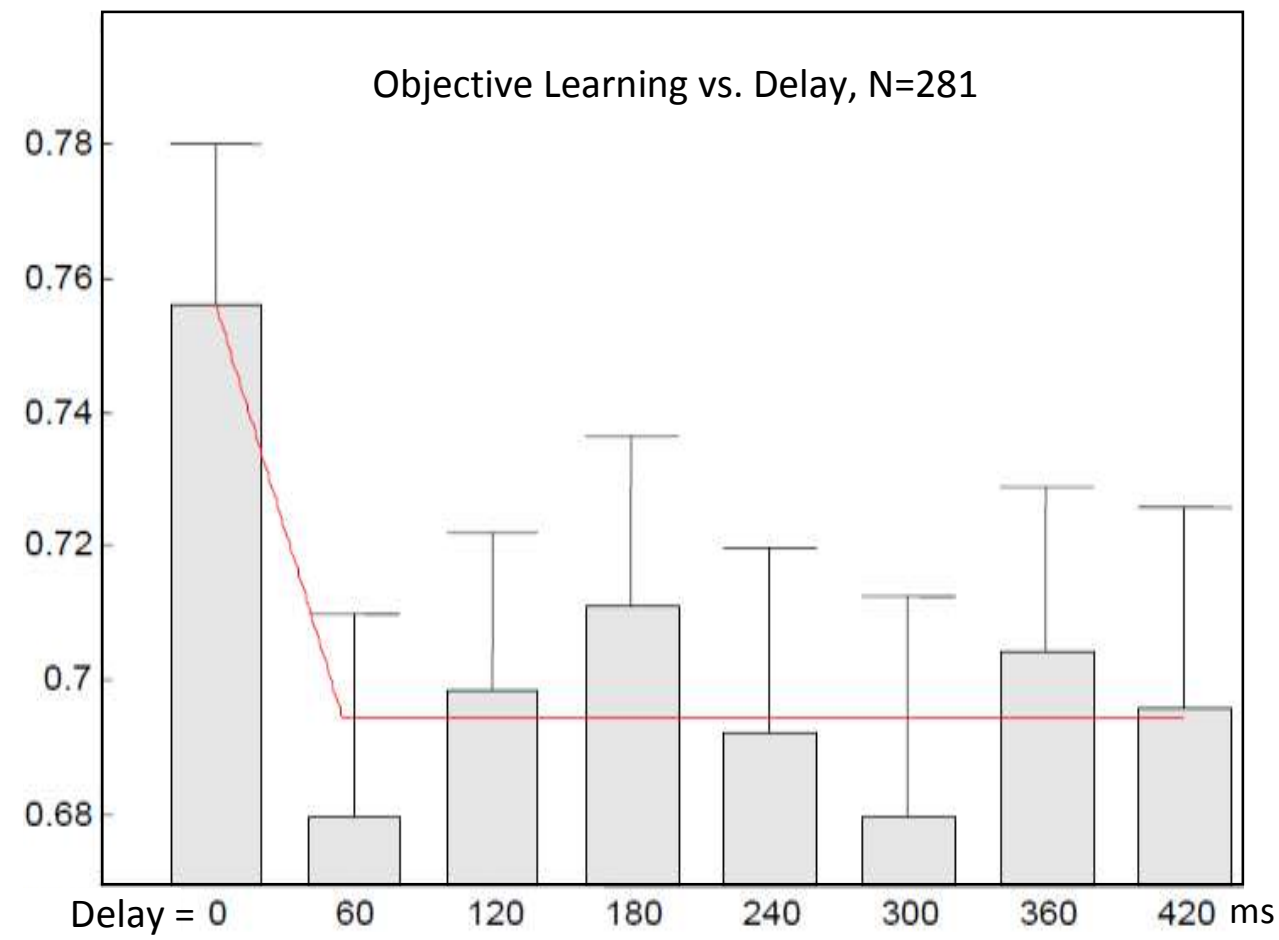

Figure 1: Objective learning scores from the original study

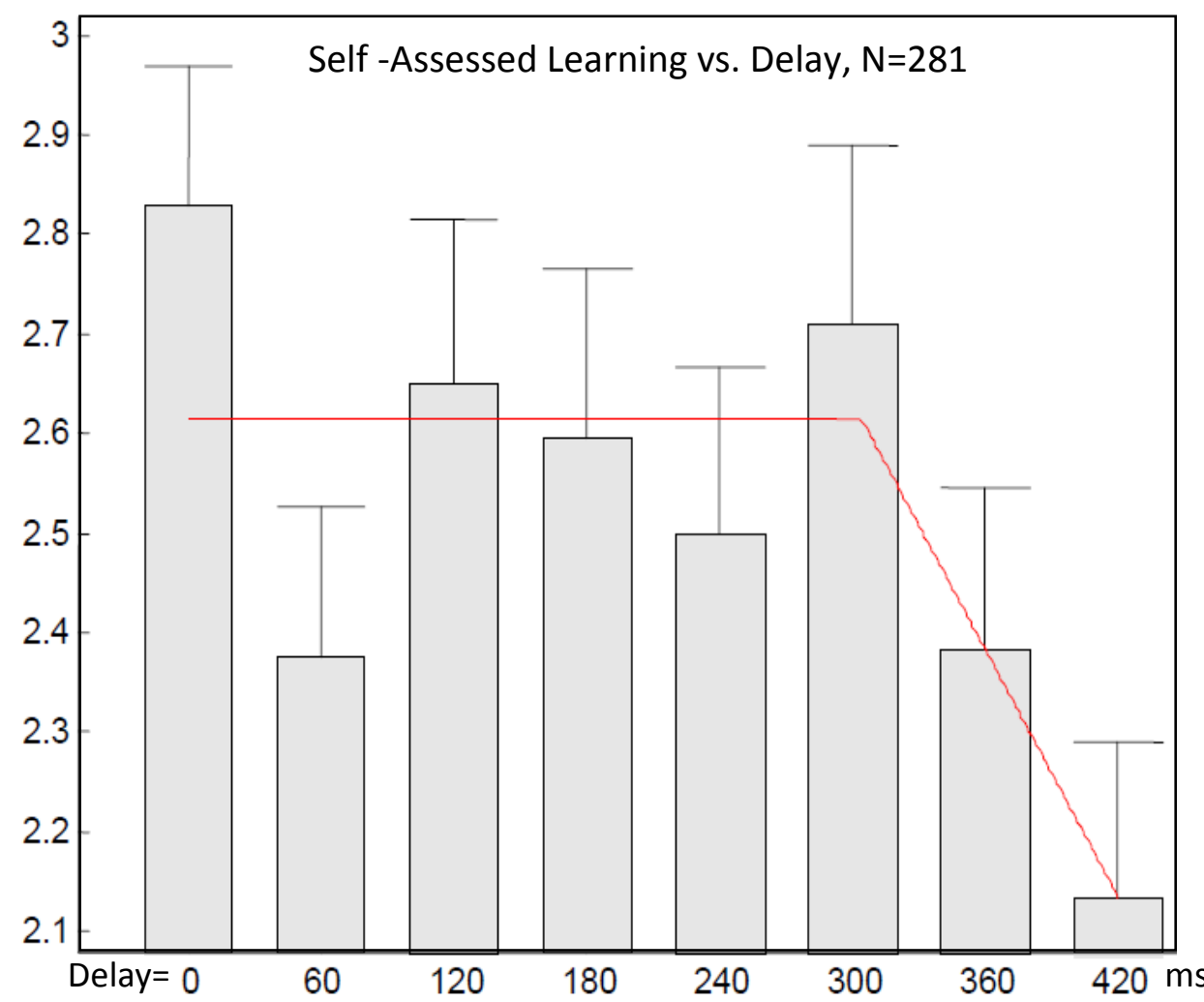

Figure 2: Self-assessed learning scores from the original study 


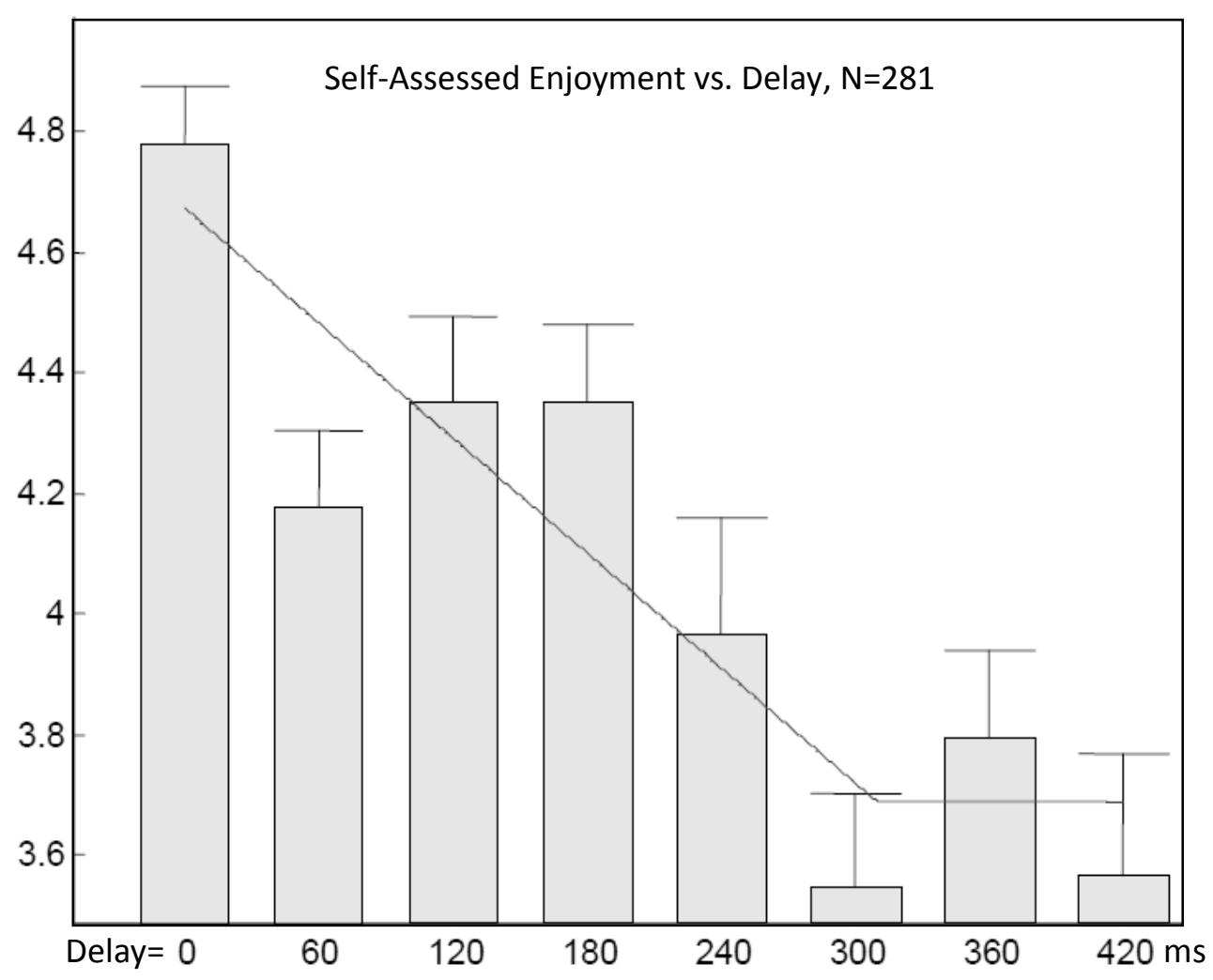

Figure 3: Self assessed enjoyment scores from the original study

\section{Results of the Longitudinal Study:}

While the initial study of 281 students provided a snapshot of the effects of network delays on learning, it did not address how students learning characteristics and tolerance for delay evolved over time. As a follow on to the original study, a longitudinal study was initiated in the fall semester of 2008 with a group of 38 incoming freshmen. Students were randomly assigned one of eight versions of the Fourier synthesis tutorial and were instructed to use the interactive features of the tutorial to help them answer fill in the same questionnaire used in the original study. In the fall semester of 2011 when this group of students started their senior year, they used the identical version of the tutorial software assigned to them in 2008 to revisit the questions on Fourier analysis that they had initially answered as freshmen. As in the initial study, students' responses were processed to obtain measures of objective learning, self-assessed learning and enjoyment.

Results for the group of students taking part in the longitudinal study deviated somewhat from those generated in the original study, with the largest differences observed in the characteristics of objective learning. In the longitudinal study, freshman using the tutorial scored almost $11 \%$ lower than the average objective learning scores of students in the original study for delays of $300 \mathrm{~ms}$ or less, (see figure 4). For delays greater than $300 \mathrm{ms,}$ the freshmen in the longitudinal study experienced increases in objective learning, with objective learning scores attaining values of .78 and .70 for delays of $360 \mathrm{~ms}$ and $420 \mathrm{~ms}$ respectively. As seniors, the objective learning scores for the 38 subjects in the longitudinal study started out lower than scores for the group of students in the original study. For delays greater than $180 \mathrm{~ms}$ the objective learning scores for the seniors in the longitudinal study took on a positive trend, attaining an average value of .69 , (see figure 5). The average objective learning score from the original study for delays of $60 \mathrm{~ms}$ or more is .68 . The primary difference between the longitudinal study group and the group of students in the original study is lower performance at small delays. This phenomenon may be due in part to the small number of students available for the longitudinal study. In the longitudinal study only 4 or 5 
students took each version of the tutorial whereas in the original study, upwards of 40 students used each of the eight versions of the tutorial.

Self-assessed learning results fall more in line with the characteristics observed for the 281 student population. Initially the group of students tracked in the longitudinal study had a drop in self assessed learning for delays greater than $360 \mathrm{~ms}$, (see figure 6). After four years, this group had become less confident in their learning, as evidenced by a downward trend in self assessed learning for any delay greater than $300 \mathrm{~ms}$, (see figure 7). Selfassessed enjoyment for the longitudinal study group displays a similar trend. Initially as freshmen this group reported the largest possible enjoyment rating when delays were zero, and a minimum enjoyment rating of about 2.25 at a delay of $240 \mathrm{~ms}$, (see figure 8 ). At all other delay times, the students reported a nearly uniform enjoyment rating of 4 on the Likert scale. As seniors this group saw its peak enjoyment of 5 at $240 \mathrm{~ms}$ and a downward trend in enjoyment for any delays greater than $240 \mathrm{~ms}$, (see figure 9 ).

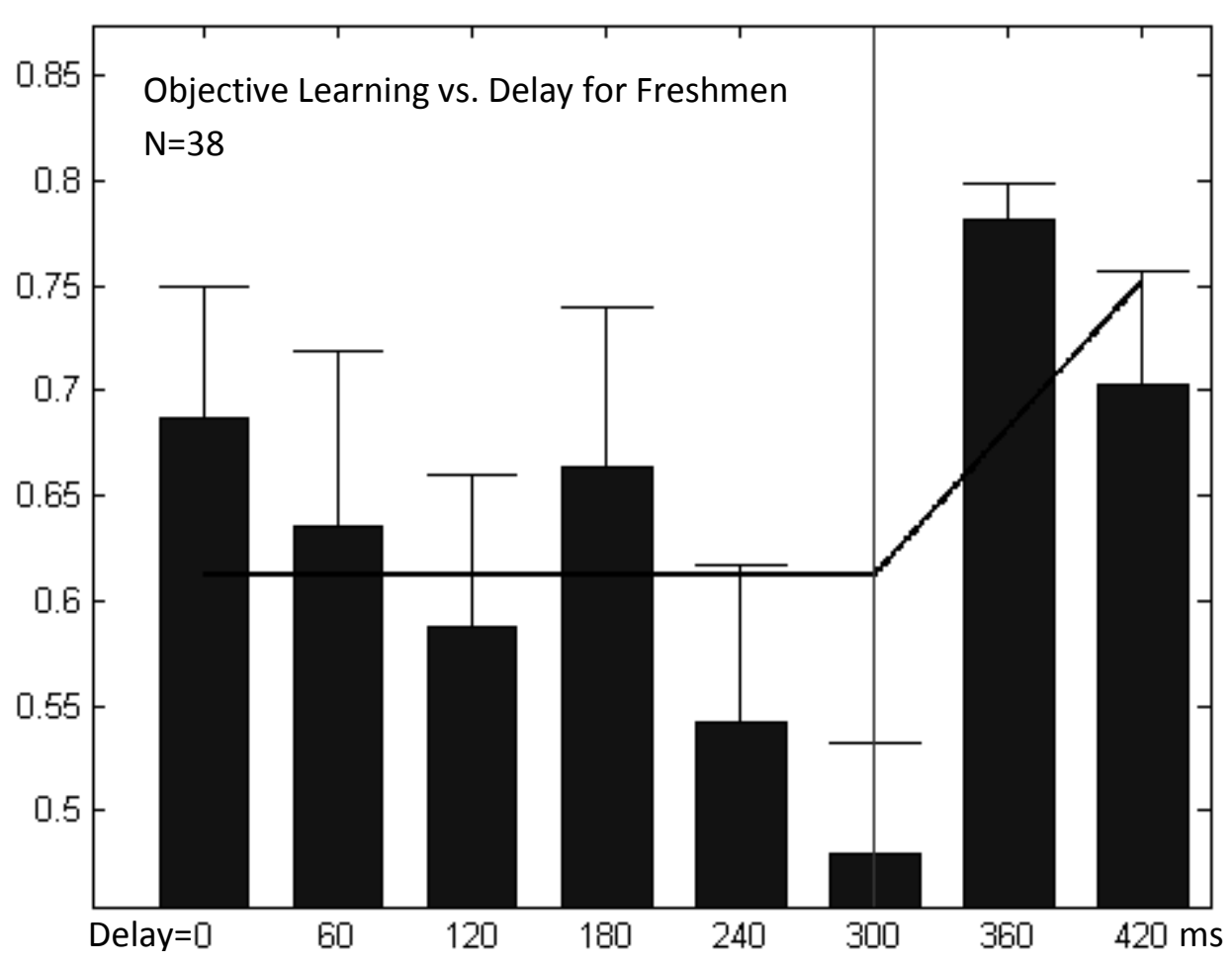

Figure 4: Objective learning scores for freshmen 


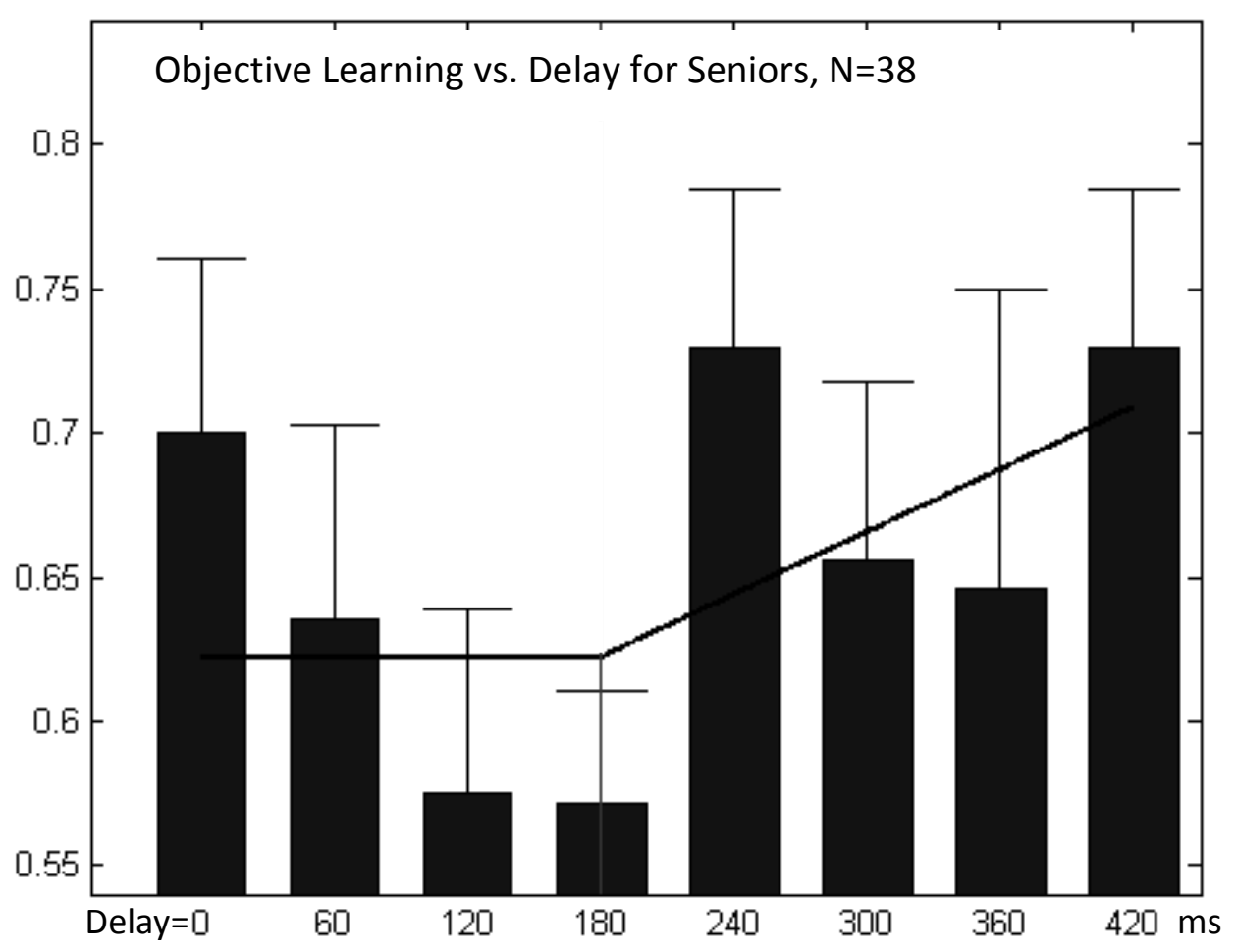

Figure 5: Objective learning scores for seniors

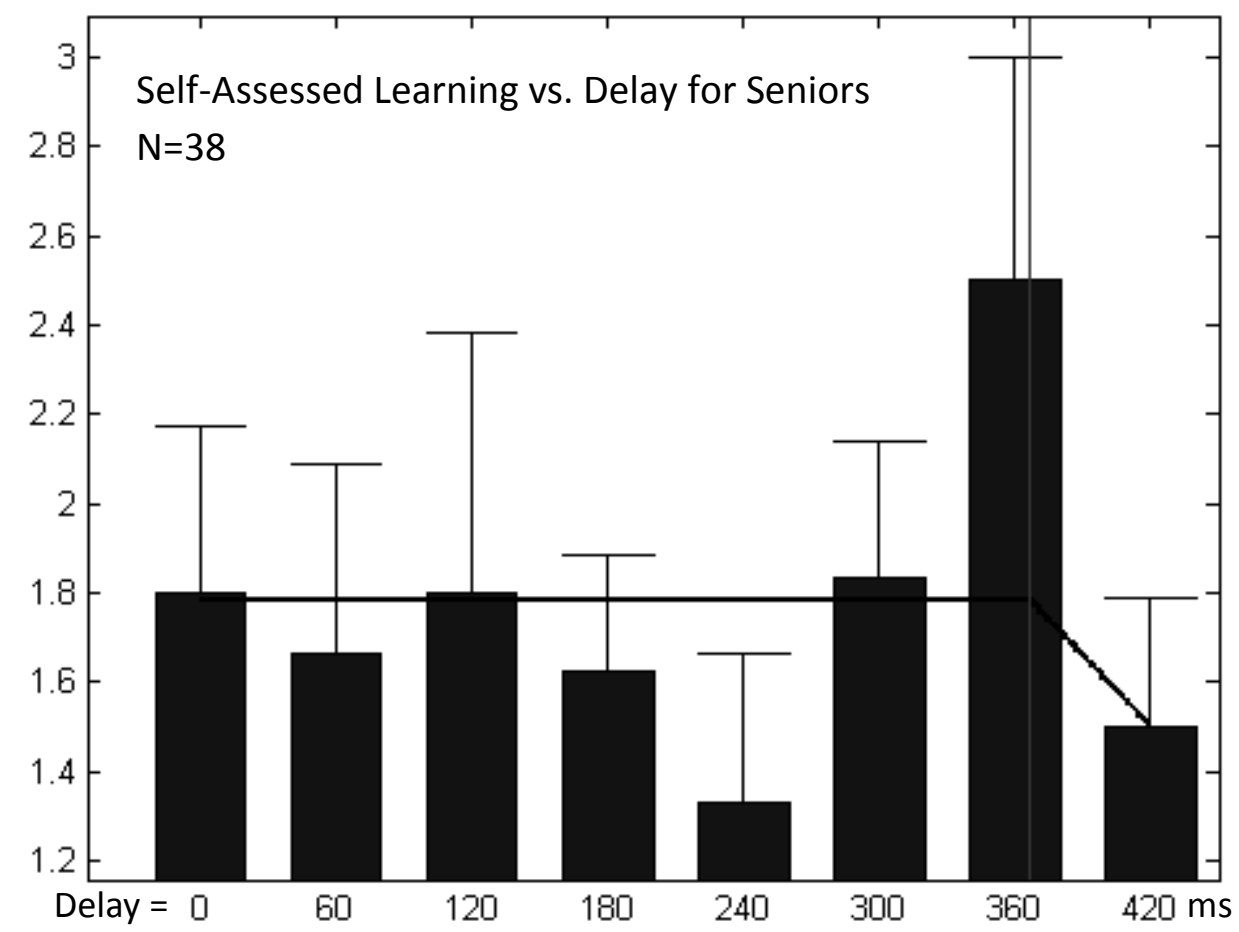

Figure 6: Self-assessed learning scores for freshmen 


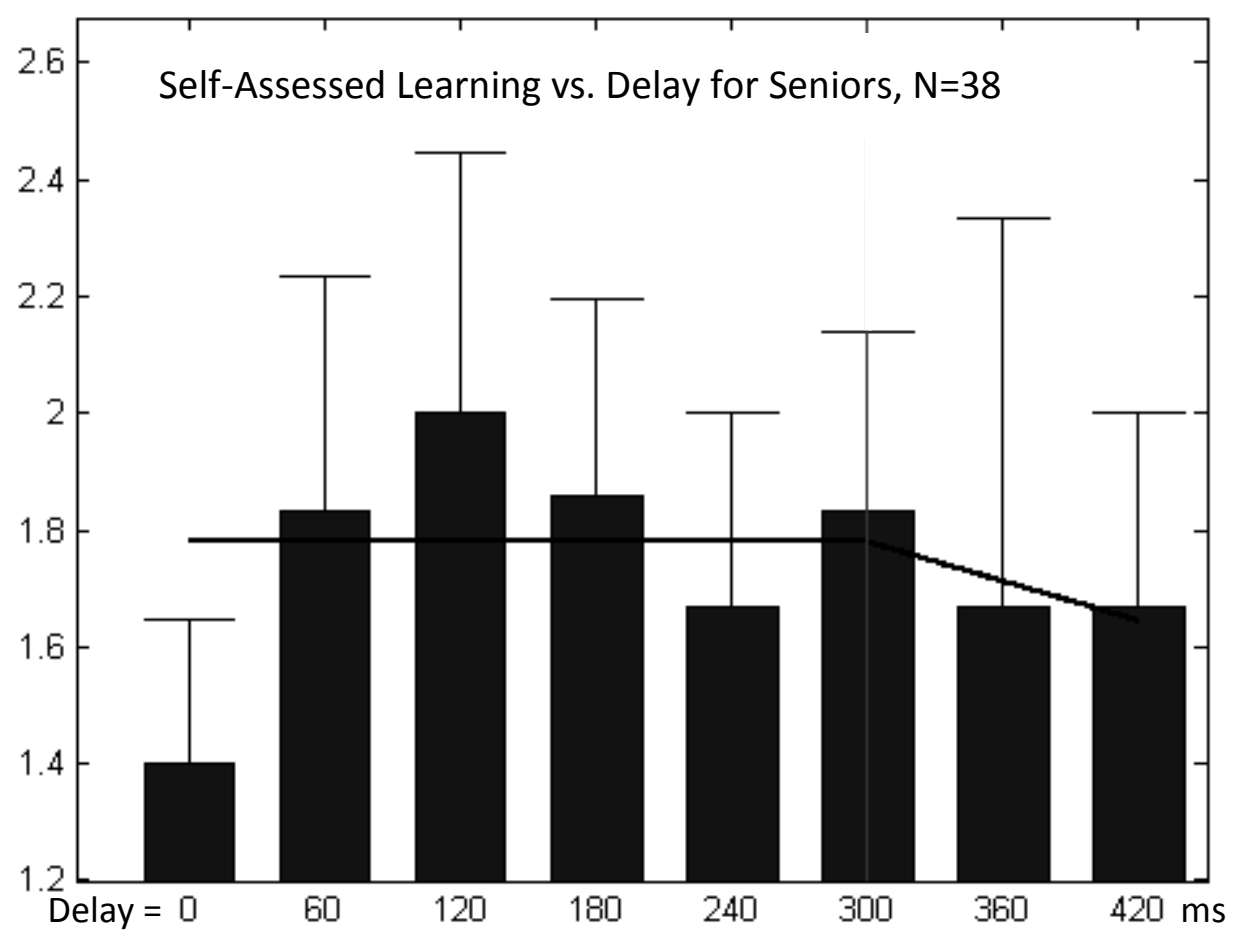

Figure 7: Self-assessed learning scores for seniors

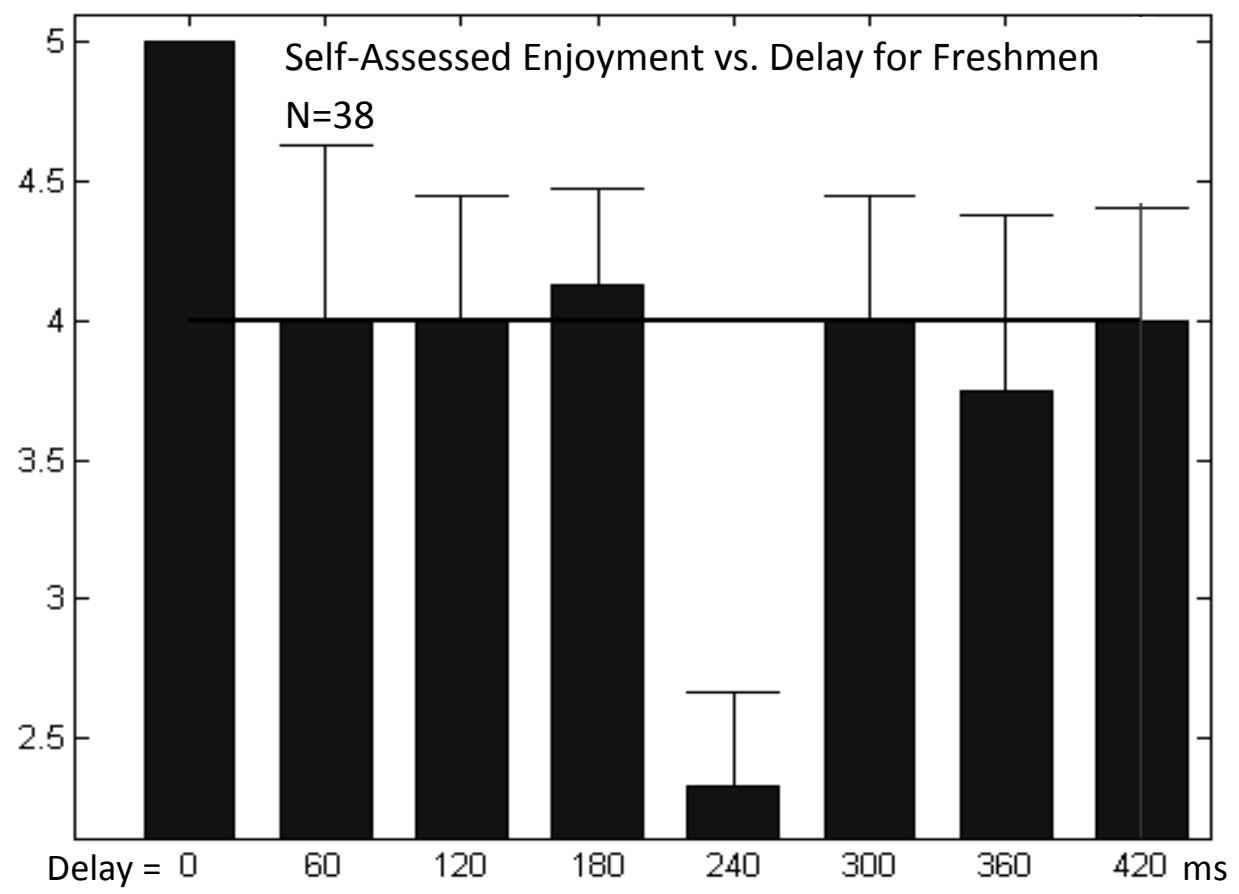

Figure 8: Self-assessed enjoyment scores for freshmen 
Self-Assessed Enjoyment vs. Delay for Seniors, $\mathrm{N}=38$

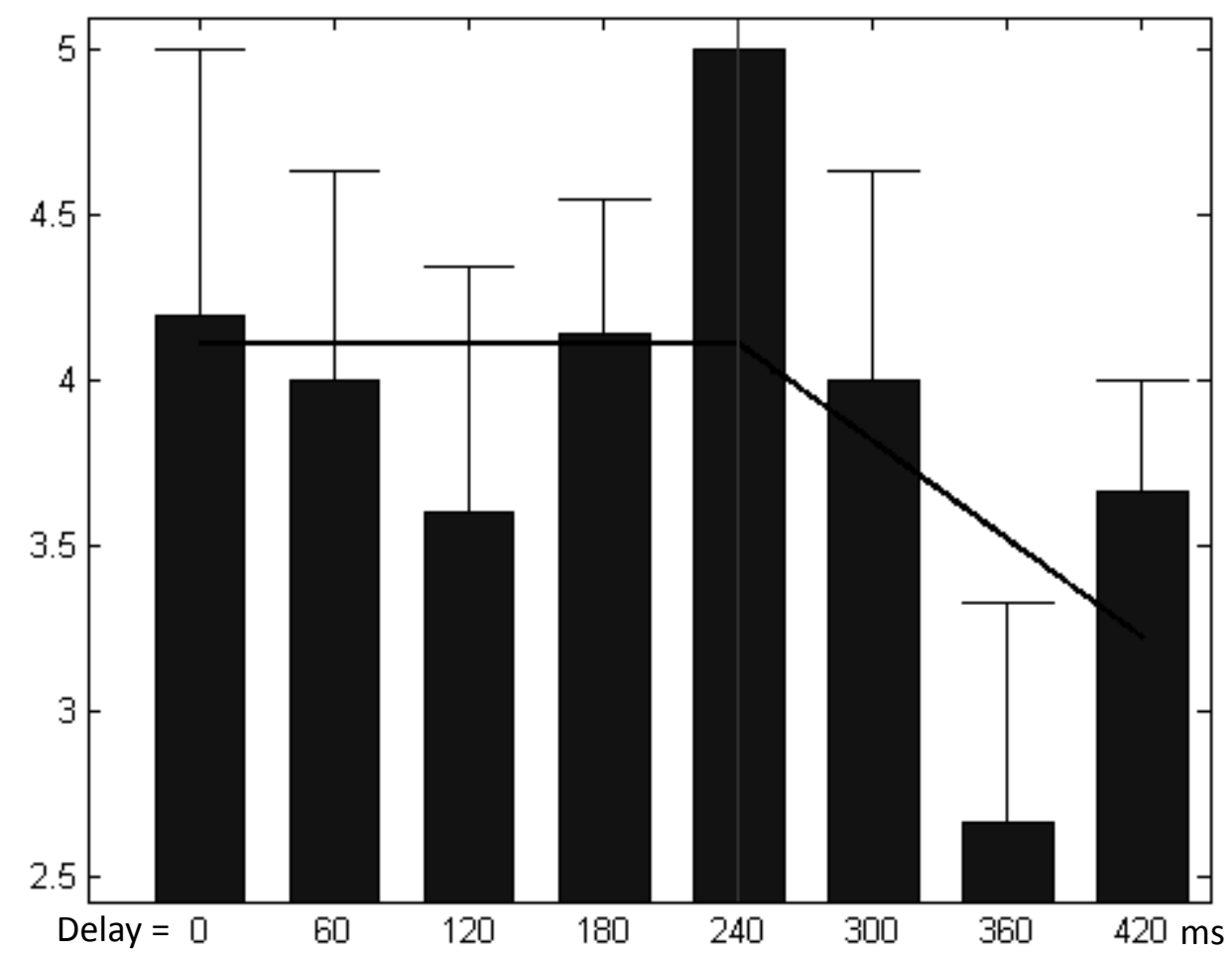

Figure 9: Self-assessed enjoyment scores for seniors

The primary goal of performing the longitudinal study was to investigate how the learning characteristics of students with respect to network delays change as students' progress from freshmen to seniors. Although some insight can be gleaned by looking at the changes of individual learning measurements, (objective learning, self assessed learning, enjoyment), for each delay time, between freshmen and seniors, it is difficult to identify useful trends. An alternative way to assess the changes in the sensitivity of the longitudinal study group to network delays is to examine students' ability for self-critique. In this work, self-critique is measured using a data space consisting of objective learning and self-assessed learning as shown in figure 10. In this plot the vertical axis consists of objective learning scores, while the horizontal axis consists of self assessed learning scores normalized from the Likert scale to a value between 0 and 1 . The purpose of the plot is to characterize the sophistication of students by seeing how closely their beliefs about what they have learned match their actual performance. Ideally, students would be able to accurately assess how well they learned material so as not to apply their new found knowledge inappropriately where potentially dangerous outcomes could be the result. Graphically, an ideal ability for selfcritique occurs when observed values of self assessed learning and objective learning lie on a line that makes a 45 degree angle with the horizontal axis of the self-critique plot. As self assessed learning and objective learning characteristics move toward the lower right hand corner of the plot, it is indicative of students who are overly confident about what they have learned. A person with this overly optimistic profile may inappropriately apply knowledge, and could even cause harm. On the other hand people with a learning profile that places them in the upper left hand side of the plot suffer from a lack of confidence and may not be able to apply what they have learned in spite of the fact that their objective learning scores are high. 


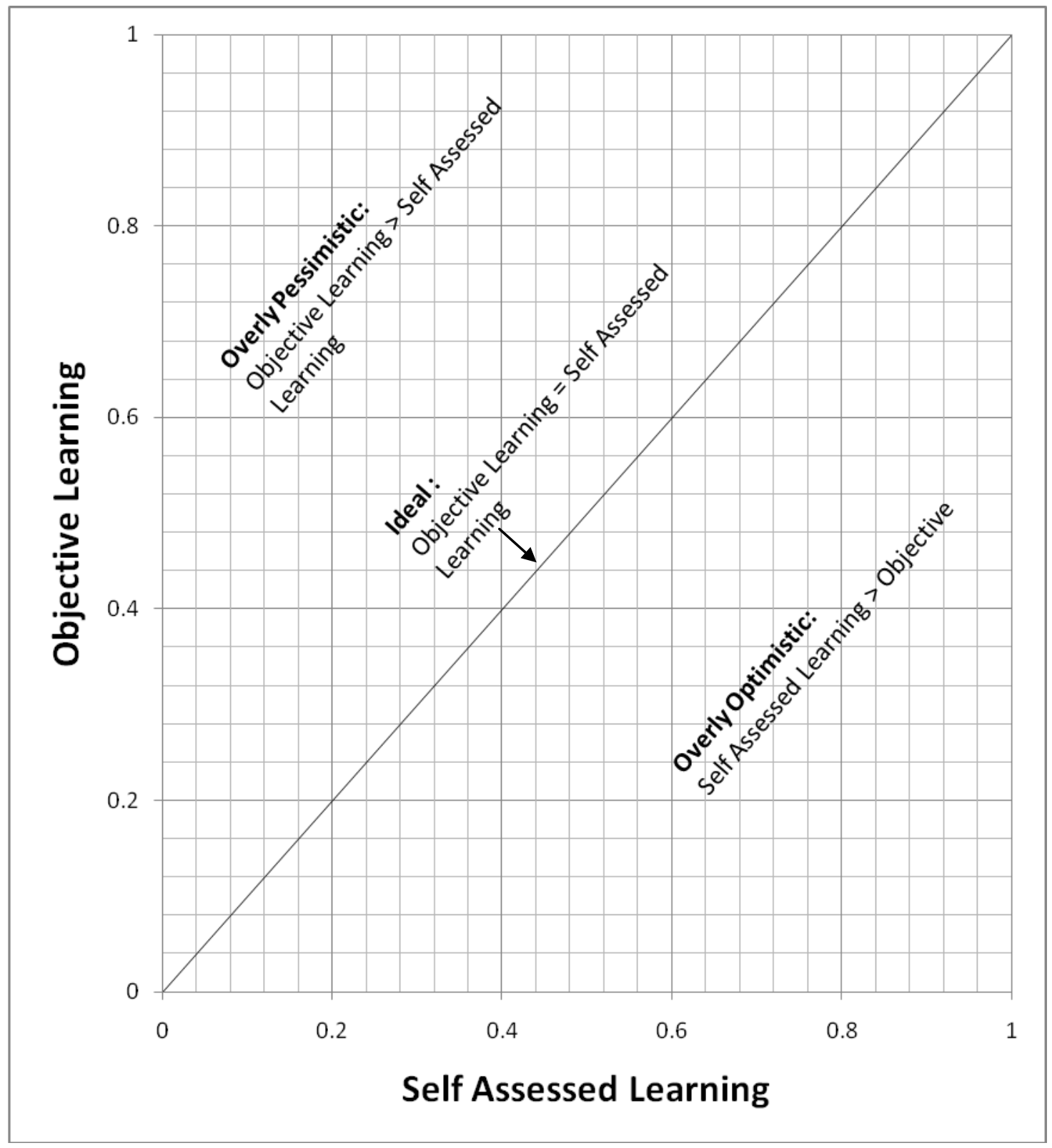

Figure 10: Self critique plot

For the purposes of this work, students' raw scores on the Fourier tutorial were plotted at the beginning of the study under the heading of "Scores as Freshmen" and at the conclusion of the study under the heading of "Scores as Seniors", (see figure 11). 


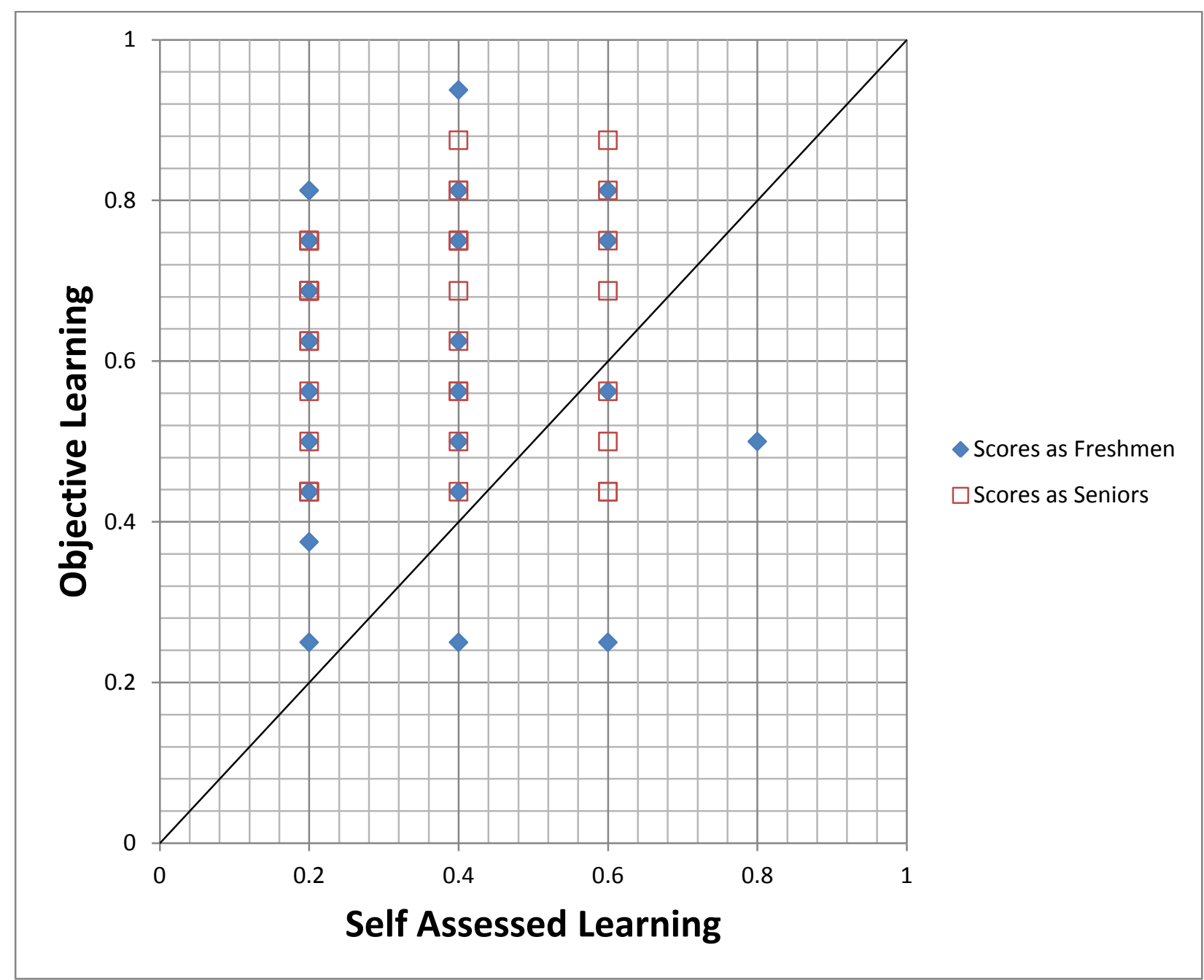

Figure 11: Self Critique plot over the longitudinal study

This plot shows all 38 of the data points from the longitudinal study and as can be seen, the data generated by the participants in the study as freshmen occupies a much larger area than the data taken when the participants were seniors, (note that some data points overlap). In addition the grouping of the freshmen data points is predominantly in the lower right hand portion of the plot, indicating an overly optimistic style of learning. There are many possible reasons for this bias including a lack of experience with mathematical concepts, and the novelty of the Fourier synthesis tutorial itself. By the time the students have reached their senior year, the grouping of data has migrated towards the upper left quadrant, and the students have evolved towards a more conservative style of learning. This shift may be due in part to more training in mathematics over the intervening years of the study, as well as the development of more critical thinking skills through experience in students' respective academic majors. In addition to the shift of the "centroid" of each data set, the variance of the data also appears to decrease as students proceed from freshmen to seniors. This is particularly important since it suggests that in parallel with the fundamental changes students are experiencing in their style of learning, they are also becoming less sensitive to the effects of delay on learning as they approach graduation. This point can be reinforced by examining the mean objective and mean self assessed learning scores for each of the eight delay times used in this study. In figure 12, each point on the plot consists of a mean value of self assessed learning and a mean value for objective learning based on all of the students that took the tutorial with a particular delay time. Delay times for each point on the plot are shown in blue for data gathered from the students as freshmen, and black for data taken from the students as seniors. Each data set is encircled in a bounding ellipse to show the relative variations on the mean score over the entire set of 8 delays. 


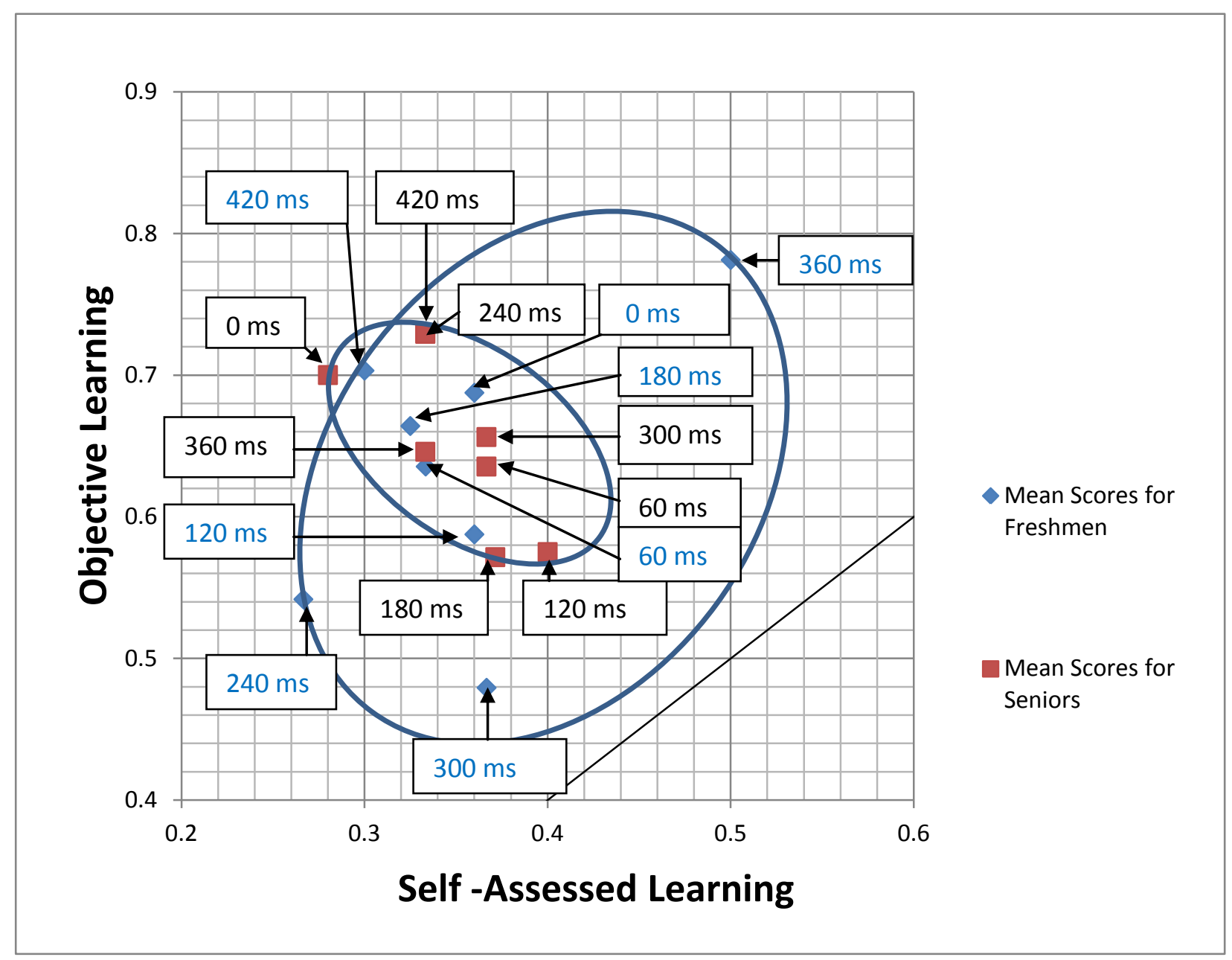

Figure 12: Self critique characteristics as a function of delay time

Based on this figure it is clear that the effect of delay time on the students as freshmen was much greater than for the same students as seniors. Mean values for objective and self assessed learning for the freshmen and senior results are fairly close together for delays up to $180 \mathrm{~ms}$. At delays of $240 \mathrm{~ms}, 300 \mathrm{~ms}$ and $360 \mathrm{~ms}$, there are large differences between data gathered when the students were freshmen data and when they were seniors. One possible explanation of this behavior is that as students accumulate experience over the course of their education, they learn coping strategies that allow them to perform consistently even in the presence of delays. Notice that even without an extensive experience set at their disposal, freshmen who took the tutorials with a delay of $180 \mathrm{~ms}$ or less performed at a similar level to seniors.

\section{CONCLUSIONS}

In this work a longitudinal study of the effects of network delays on learning was performed using a Fourier Synthesis tutorial on a group of 38 students that initially used the tutorial as freshmen and then re-took the tutorial in their senior years. In addition to objective learning, self assessed learning and self assessed enjoyment measurements to determine the effective of delays on learning, self-critique characteristics of the students in the study were also compiled. Based on the self critique characteristics plotted for data taken from the students as freshmen and then as seniors, two major results were identified: 
- $\quad$ As freshmen, students in the study tended to be "overly optimistic" about what they learned, not always realizing that they had not mastered a concept. As seniors, students in the study took on a more conservative stance and were in general less confident about their learning even when objective learning scores were relatively high.

- $\quad$ As seniors, students in the study were less sensitive to network delays than they were as freshmen.

Together these results imply that experience plays a large part in the development of learning styles and the ability to work in a non-ideal environment, (i.e. significant network delays). Additional work with larger sample sizes is needed to better model the interaction between experience levels, network delays, and the resulting learning outcomes.

\section{AUTHOR INFORMATION}

Dr. Jay Sullivan, Associate Professor of Mechanical Engineering at the Virginia Military Institute, received his B.S.M.E. from the University of Vermont and his Ph.D. from Rensselaer Polytechnic Institute. He has held teaching positions at the University of Michigan-Dearborn, and the University of Vermont. Prior to joining the faculty at the Virginia Military Institute in the fall of 2004, he was employed by JMAR Inc. where he was involved in research and development of X-ray lithography systems for the semiconductor industry. E-mail: sullivanga@vmi.edu

Dr. H. Francis Bush a Professor of Economics and Business at the Virginia Military Institute. He received a B.A. in Mathematics from the State University of New York at Buffalo, NY, his Masters of Accountancy from The Ohio State University and his PhD from the University of Florida. The focus of his doctoral work was human information processing and is currently finishing studies related to Enron-Anderson. At VMI he teaches Principles and Intermediate Accounting, Financial Statements Analysis, and Statistics. E-mail: bushhf@vmi.edu (Corresponding author)

Dr. James Squire is an Associate Professor of Electrical Engineering at the Virginia Military Institute. He received a B.S. in Electrical Engineering from the United States Military Academy in West Point, NY and served in the army as a Military Intelligence officer during Desert Storm. Although his $\mathrm{PhD}$ is in electrical engineering, he completed his doctoral work in a biomedical engineering laboratory at MIT and has interests in analog and digital instrumentation, signal processing, biomechanics, patent litigation, and cardiology. At VMI he teaches analog circuitry, continuous time and discrete time signal processing, and advises a variety of independent study projects. E-mail: squirejc@vmi.edu

Dr. Vonda K. Walsh is a Professor of Mathematics at Virginia Military Institute. She received her B.S. in Mathematics from the University of Virginia's College at Wise, her M.S. in Pure Mathematics from Virginia Tech and her Ph.D. in Biostatistics from the Medical College of Virginia /Virginia Commonwealth University School of Medicine. E-mail: walshvk@vmi.edu

\section{Bibliography}

1. Allen, I.E., and Seaman, J., "Staying the Course: Online Education in the United States, 2008", Retrieved from http://www.sloan-c.org/publications/survey/staying course

2. Allen, I.E., and Seaman, J., "Staying the Course: Online Education in the United States, 2011", Retrieved from http://www.onlinelearningsurvey.com/reports/goingthedistance.pdf

3. Bush, F., Squire, J.C., Sullivan, G.A., Walsh, V. K., English, A., and Bolen, R., “ An Investigation of the Effect of Network Latency on Pedagogic Efficacy: A Comparison of Disciplines”, Contemporary Issues in Education Research 2008, Vol. 1, No. 4, pp. 11-26

4. Dollar, A., and Steif, P.S., "Enhancing Traditional Classroom Instruction With a Web-Based Statics Course", 2007 Frontiers in Education

5. $\quad$ Parsad, B, and Lewis, L., "Distance Education at Degree Granting Post-Secondary Institutions: 20062007”, Institute of Education Sciences, Retrieved from http://nces.ed.gov/pubs2009/2009044.pdf 
6. Reisel, John R., Jablonski, M., Hanson, E. and Hosseini, H., "Evaluation of Factors Affecting the Success of Improving Math Course Placement for a Summer Bridge Program", Proceedings of the ASEE 2010 Annual Conference, Louisville Ky.

7. Squire, J.C., Bush, H.F., Walsh, V.K., Sullivan., G.A., and English, A., "Results From a Multi-Center Investigation of the Effects of Network Latency on Pedagogic Efficiency", Computers in Education Journal, 18:4 (2008), pp.103-112

8. Walsh, V.K., Bush, F., Squire, J.C., Sullivan, G.A., “A Multicenter Study of Students' Sensitivity to Screen-Update Delay", Contemporary Issues in Education Research, June 2011, Vol. 4, No 6, pp. 7-14 
NOTES 